Nonlin. Processes Geophys., 20, 41-45, 2013

www.nonlin-processes-geophys.net/20/41/2013/

doi:10.5194/npg-20-41-2013

(C) Author(s) 2013. CC Attribution 3.0 License.

\title{
Quantifying soil complexity using network models of soil porous structure
}

\author{
M. Samec ${ }^{1}$, A. Santiago ${ }^{2}$, J. P. Cárdenas ${ }^{2}$, R. M. Benito ${ }^{2}$, A. M. Tarquis ${ }^{3}$, S. J. Mooney ${ }^{4}$, and D. Korošak ${ }^{1,5}$ \\ ${ }^{1}$ Faculty of Civil Engineering, University of Maribor, Smetanova ulica 17, 2000 Maribor, Slovenia \\ ${ }^{2}$ Grupo de Sistemas Complejos, Departamento de Física, Universidad Politécnica de Madrid, 28040 Madrid, Spain \\ ${ }^{3}$ CEIGRAM ETSIA, Universidad Politécnica de Madrid, 28040 Madrid, Spain \\ ${ }^{4}$ Environmental Sciences Section, School of Biosciences, Sutton Bonington Campus, University of Nottingham, \\ Loughborough, Leics LE12 5RD, UK \\ ${ }^{5}$ Institute of Physiology, Faculty of Medicine, University of Maribor, Maribor, Slovenia
}

Correspondence to: M. Samec (marko.samec@um.si)

Received: 23 July 2012 - Revised: 16 December 2012 - Accepted: 19 December 2012 - Published: 15 January 2013

\begin{abstract}
This paper describes an investigation into the properties of spatially embedded complex networks representing the porous architecture of soil systems. We suggest an approach to quantify the complexity of soil pore structure based on the node-node link correlation properties of the networks. We show that the complexity depends on the strength of spatial embedding of the network and that this is related to the transition from a non-compact to compact phase of the network.
\end{abstract}

\section{Introduction}

One of great challenges in soil science today (Lal, 2007) is to understand how soils behave as a complex system (Crawford, 2010). Over the last decade, network theory has become a much discussed cross-disciplinary research field contributing to social, biological and information sciences (Barabasi, 2009, 2012), demonstrating that quite diverse systems might share similar topological organization. Whilst soil scientists have studied the complexity of soil pore space for decades (Berkowitz and Ewing, 1998), it is only recently that soil pore networks have been considered as complex systems. Specifically two approaches to describe soil pore structure as complex networks of pores have recently been proposed (Mooney and Korošak, 2009; Cárdenas et al., 2010), both emphasizing the role of the spatial embedding of the network. Soil pore structure was described as a complex network of pores using a spatially embedded varying fitness network model (Mooney and Korošak, 2009) or heterogeneous preferential attachment scheme (Santiago et al., 2008). Both approaches reveal the apparent scale-free topology of soils with a power-law distribution $P(k) \propto k^{-\gamma}$ of the degrees $k$ of nodes as a consequence of a heterogeneous soil pore size distribution.

\section{Methodology}

Soil pore networks are spatial networks (Barthelemy, 2011), i.e. complex networks with nodes (pores) embedded in space. Often the interaction $T_{i j}$ between distant nodes $i$ and $j$ is modelled using a gravity model:

$T_{i j}=s_{i} s_{j} f\left(d_{i j}\right)$

where $f$ describes the effect of spatial embedding of the network. In the threshold soil pore network (Mooney and Korošak, 2009) model, $f=d_{i j}^{-m}$ and the nodes are linked if $T_{i j}>\theta$. Here, the non-dimensional parameter $m$ measures the importance of the distance, and the threshold value $\theta$ controls the number of links in the network. In the growing network mechanism with extended preferential attachment rule (Santiago et al., 2008), the probability to connect depends on the pore size $s_{i}$ and the distance between the pores $i$ and $j$ to the power of $m$.

In the threshold model the scaling exponent is for strong enough embedding with $m>D /(\alpha-1)$ given by $\gamma=1+$ $m(\alpha-1) / D$ (Masuda et al., 2005; Yakubo and Korošak, 2011), where $D$ is the embedding or fractal dimension, while 
in the growing network model multiscaling (Bianconi and Barabasi, 2001) was found with $\gamma=1+2 / w$ (Cárdenas et al., 2010), where $w$ is the normalized fitness of the nodes. The analysis of the scale-free network embedded in fractal space (Yakubo and Korošak, 2011) has shown that we can distinguish three phases of embedded network: (i) noncompact phase for $m<m_{c 0}=D /(\alpha-1)$ with $y=2$, (ii) intermediate phase for $m_{c 0}<m<m_{c 1}=(D+1) /(\alpha-1)$, and (iii) compact phase for $m>m_{c 1}$.

Here, we first show using soil pore space structure data from 2-D X-CT soil image that both methods of network construction, in which we can tune the network heterogeneity, predict topologically similar networks of soil pore structures. Furthermore, we calculate the assortativity $r$ (Newman, 2002) as a function of network heterogeneity and find that the crossover from disassortative to assortative network configuration might depend on soil fractal properties (Rieu and Sposito, 1991; Dimri, 2000) obtained from multifractal analysis of soil structural images derived from X-ray computed tomography (Mooney and Morris, 2008).

Finally, we use the entropy of node-node link correlations (Claussen, 2007) to quantify the complexity of soil porous architecture allowing us to relate the scaling exponent of the pore size distribution and the complexity of the network of soil porous structure. This is a computationally simple network complexity measure that is sensitive to the network structure. The complexity measure is defined as the entropy of node-node link correlations that are given with the matrix elements $c_{k, k^{\prime}}$ counting the number of links between nodes with degrees $k$ and $k^{\prime}$ in the network:

$c_{k, k^{\prime}}=\left\{\begin{array}{c}\sum_{i, j=1}^{N} a_{i j} \delta_{k_{i} k} \delta_{k_{j} k^{\prime}} k \neq k^{\prime} \\ \frac{1}{2} \sum_{i, j=1}^{N} a_{i j} \delta_{k_{i} k} \delta_{k_{j} k^{\prime}} k=k^{\prime}\end{array}\right.$,

where $a_{i j}$ are the elements of the adjacency matrix. The probability that a randomly chosen edge links two nodes with the degree difference $\Delta k, b_{\Delta k}$ is then constructed from the nodenode link correlation matrix $c_{k, k^{\prime}}$ :

$b_{\Delta k}=\frac{\sum_{k=1}^{k_{\max }-\Delta k} c_{k, k+\Delta k}}{\sum_{\Delta k=0}^{k_{\max }-1} \sum_{k=1}^{k_{\max }-\Delta k} c_{k, k+\Delta k}}$.

Here the denominator is equal to $M$ (total number of edges) and the numerator gives the number of edges connecting node pairs with the degree difference $\Delta k$ for any two $k, k^{\prime}$. Finally, the complexity is defined as an entropy measure of $b_{\Delta k}$ and measures how widely the degree differences of connected node pairs in the network are distributed:

$h=-\sum_{\Delta k=0}^{k_{\max }-1} b_{\Delta k} \log b_{\Delta k}$.
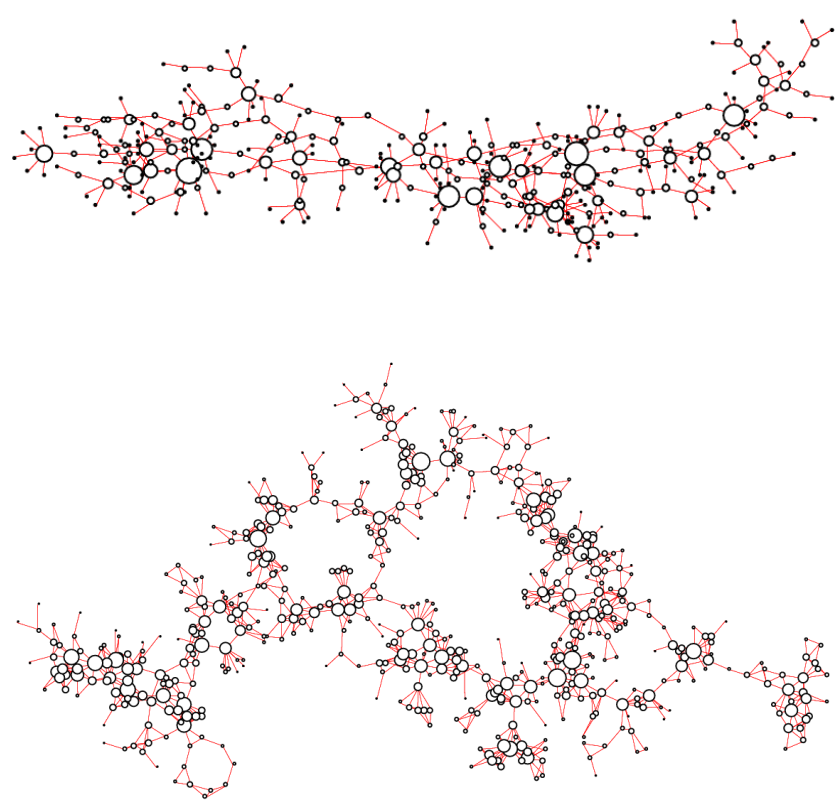

Fig. 1. Network representation of soil pore structure obtained by the evolving network (EN) model (top) and static network (SN) model (bottom) from the same set of data derived from images of soil structure and with equal model parameters: equal number of links and $m \approx 1$. The area of node corresponds to the node degree, i.e. the number of links attached. The data for the network construction included sizes and positions of the pores detected from 2-D X-CT scanned and analysed soil images. Though here rendered differently, with positions of the nodes not corresponding to actual positions of the pores in the analysed images (for networks overlayed over soil images, see Mooney and Korošak, 2009), both networks actually show similar topology with the well-connected nodes (larger dots) not directly linked, but preferably linked through nodes with small degrees (smaller dots). This type of network structure indicates that both networks are correlated.

\section{Results and discussion}

In Fig. 1 we display examples of networks representing the soil pore structure. The input data for both network models were the geometric centres of pore positions (nodes of the network) and pore sizes (measured as pore area) obtained from 2-D X-CT soil image. The number of pores obtained from image analysis was of the order of $N \approx 10^{3}$. The upper network in Fig. 1 is obtained using the growing network mechanism with extended preferential attachment rule (Santiago et al., 2008), while the lower network in Fig. 1 results from the threshold model (Mooney and Korošak, 2009). In all analysed samples the pore size distributions were found to follow a power law $W(s) \propto s^{-\alpha}$ with the scaling exponent $1<\alpha<2$. Both methods lead to scalefree organization of pore networks for small enough $m$ (of the order of 1 or less, in non-compact phase) with the degree distribution $P(k) \propto k^{-\gamma}$, and to more compact, homogenous 
Table 1. Complexity $h$ of several soil pore structures with different scaling exponents of their pore size distributions at $m=m_{c} / 2$. Pore space is black.

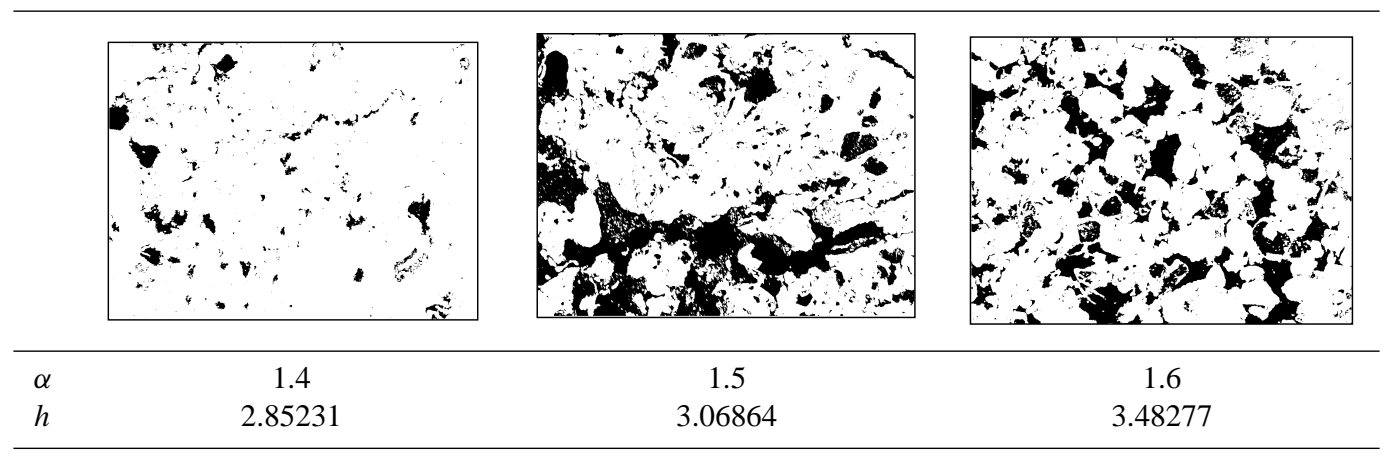

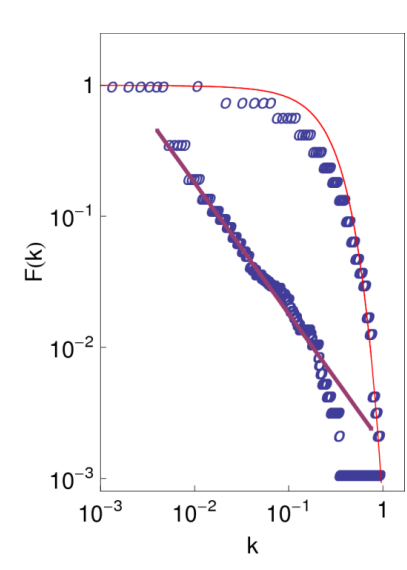

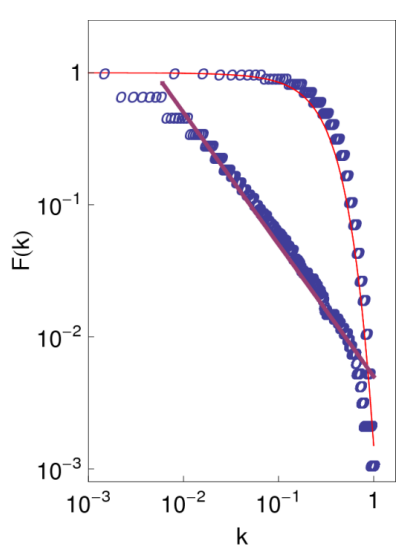

Fig. 2. Cumulative degree distributions of evolving network (EN) model (left) and static network (SN) model (right) for small and large values of the parameter $m$. The scaling exponent of the scalefree degree distribution obtained for small $m$ (straight line in plots) is $\gamma=2$. The fits are power laws (straight lines) and cumulative Poisson distributions.

organization for large $m$ as shown in Fig. 2 by the cumulative degree distributions for $m \approx 1$ and $m \gg 1$.

Multifractal analysis of the same 2-D soil images (Stanley and Meakin, 1988; Perrier et al., 2006) was performed, which yielded $D=D_{q}(q=0) \approx 1.74$ shown in Fig. 3. In an attempt to correlate the properties of the threshold network structure with the porous structure as obtained from 2-D soil image, we considered the assortativity $r$ (Newman, 2002), which is the Pearson's correlation coefficient of the degrees of adjacent nodes, of the threshold network as a function of the parameter $m$.

From the result displayed in Fig. 4, we see that there is a crossover from disassortative $(r<0)$ to assortative $(r>0)$ network structure at approximately $m_{c} \approx 4.3$. This change is a consequence of progressively more homogeneous network structure with increasing $m$. In geographical scale-free networks this crossover was found to occur when the degree scaling exponent was $\gamma=3$ (Morita, 2006). Equating

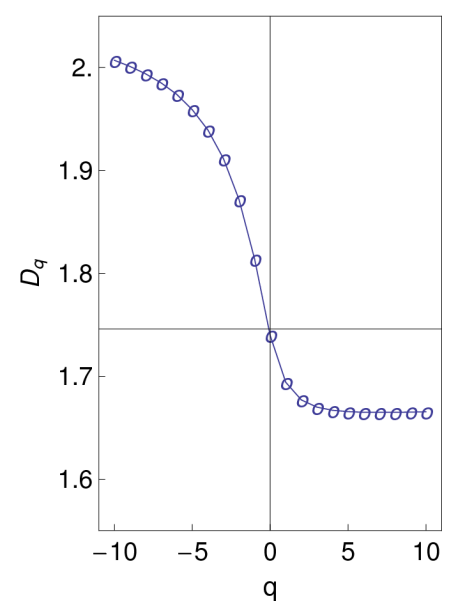

Fig. 3. Results of the multifractal analysis of the binary soil image. Fractal dimension as a function of scale parameter $q$ is shown.

the scaling coefficients for the growing network and threshold network models gives the crossover parameter $m_{c}=$ $2 D /(\alpha-1)=2 m_{c 0}>m_{c 1}$ indicating that the crossover from disassortative to assortative network occurs well within in the compact phase of the soil network. The degree scaling exponent in the growing network model exhibits multiscaling $\gamma=1+2 / w$, so a homogenous network with normalized fitness sharply distributed around $w=1$ will have $\gamma=3$.

To further explore the effect of the network parameter $m$ on node correlations, we calculated the node-node link correlations $c_{i j}$ (Claussen, 2007) defined as links from node with degree $k_{i}$ to node with degree $k_{j}$.

We expect a soil pore network with a large $h$ to have many links that connect nodes of various degrees, while networks with low $h$ will have mostly links connecting node pairs of almost the same degree resembling a lattice-like network or a network close to a complete graph.

The correlation matrices for a smaller geographical threshold network are shown in Fig. 5 for $m \ll 1$ (a), $m=1$ (b) and $m \gg 1$ (c). Again, we illustrate that, with the increasing value 


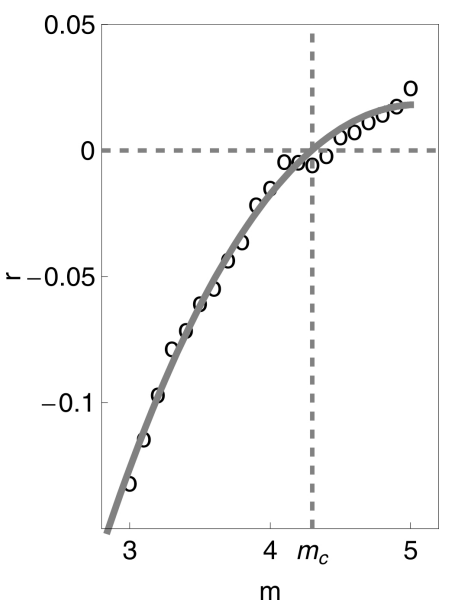

Fig. 4. Pearson's coefficient $r$ applied to the links in the network as a measure of degree-degree correlations. $r$ is shown as a function of parameter $m$ in the static network model. We observe a crossover from disassortative $(r<0)$ to assortative $(r>0)$ network structure at $m_{c}=4.3$.

of the parameter $m$, the network changes from disassortative (nodes with different degrees preferably connected) to assortative type.

To quantify the complexity $h$ of the soil pore structure represented with the network, we computed the entropy of the normalized diagonal sums of the correlation matrix $c_{i j}$. Finally, we have calculated the complexity of several soil pore structures with different scaling exponents of their pore size distributions at $m=m_{c} / 2$ (Table 1).

In the following we discuss two possible geophysical implications of our findings that may help to elucidate the role of the threshold network model parameter $m$ : the scaling of fractures in rocks (Berkowitz et al., 2000) and the influence of the pore network properties on biological invasion in soil (Perez-Reche et al., 2009, 2012).

The study of fracture networks in rocks (Berkowitz et al., 2000) showed that the length distribution of fractures in rocks scales as $n(l) \propto l^{-a}$. Here $a$ is the fracture length distribution exponent. For $a>D+1$ the scaling properties are independent of system size; for $a<D+1$ they depend on the system size, and $a=D+1$ signals the connectivity threshold ( $D$ is here the fractal dimension of the problem). In experiments they find that the range of $a$ is $1<a<3$. If we compare $a$ to the scaling exponent of length distribution function for scale-free networks embedded in fractal space (Yakubo and Korošak, 2011), a model we used to describe soil pore networks above, we have $a=1-D+m(\alpha-1)$. Immediately we get that the connectivity threshold $a=1+D$ corresponds exactly to $m_{c}=2 D /(\alpha-1)$, the suspected crossover from disassortative to assortative organization of the network. Furthermore, we have the lower bound $a=1$ occurring at $m_{c 0}=D /(\alpha-1)$, i.e. at the boundary between the noncompact and the intermediate phase of the network structure. a) $m \ll 1$

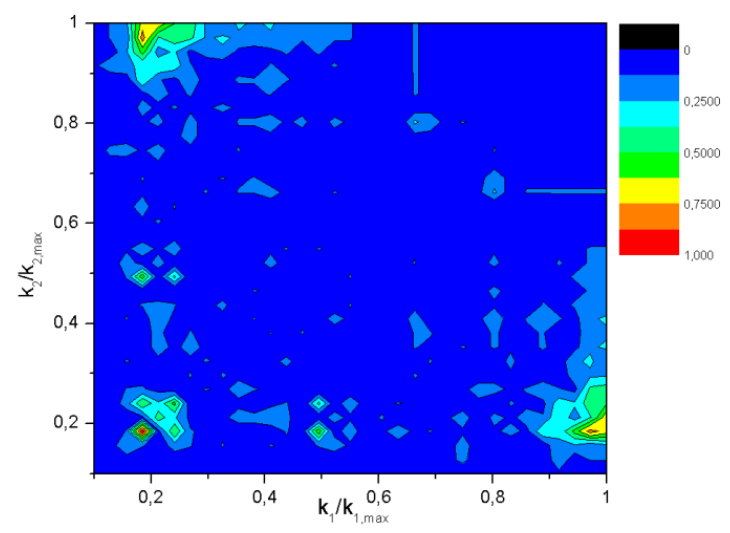

b) $m=1$

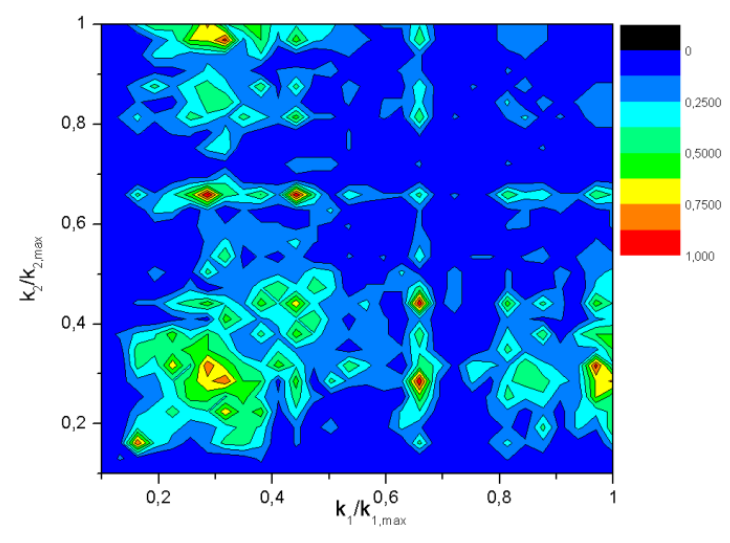

c) $m \gg 1$

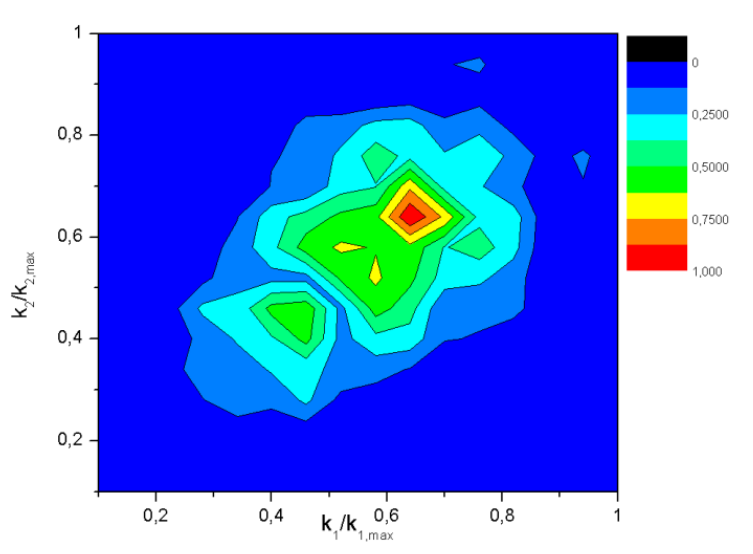

Fig. 5. Correlation matrices for a smaller geographical threshold network. 
The embedding parameter $m$ of the threshold soil pore network model controls the compactness of the network organization. For values $m<m_{1}$ (non-compact and intermediate phase) the soil pore network exhibits long range connections between pores, while in the compact phase $m>m_{1}$ the pores in the network are mostly connected to spatially nearest neighbours. Recently, the impact of the structural heterogeneity of soil pore networks on microbial spread in soils has been investigated using soil pore network models (PerezReche et al., 2009, 2012), and the idea of long range bridges that link distance pores has been introduced to explain the strong effect of the pore channel size heterogeneity on microbial invasion in soils. The pore channel size was found to be correlated with the arc length of the pore channel between two pores $i$ and $j$ as $R_{i j} \propto L_{i j}^{-\beta}$ (Perez-Reche et al., 2009). It was also suggested that the pore channel size depends on the radii of pores $i$ and $j: R_{i j} \propto R_{i} R_{j}$ (Li et al., 1986). Bringing these observations together gives the expression for the pore channel size $R_{i j} \propto R_{i} R_{j} L_{i j}^{-\beta}$ that is similar in structure to the interaction $T_{i j}$ (see Eq. 1) used to construct the soil pore network model. However, the implications of the possible connection between the spatial embedding of the soil pore network and the invasion of microorganisms in soils remain to be further investigated.

In conclusion, soil pore networks obtained from two different models demonstrate their scale-free structure, but with characteristics such as assortativity or node-node link correlations that depend on the strength of spatial embedding of the network. For the evolving soil network model (Santiago et al., 2008, Cárdenas et al., 2010, 2012), it was shown that the scaling exponent of the asymptotic degree distribution is within the interval $1<\gamma \leq 3$. Comparing the two network models, this finding sets the upper limit to the strength of embedding in the threshold network model $m \leq m_{c}=$ $2 D /(\alpha-1)$ indicating that soil pore networks are predominantly disassortative. We have used an entropic measure for the complexity of the soil pore network and showed that this quantity is sensitive to differences in soil pore structure as obtained from 2-D images of soil structure.

Edited by: J. Wanliss

Reviewed by: M. Small and one anonymous referee

\section{References}

Barabasi, A.-L.: Scale-Free Networks: A Decade and Beyond, Science, 325, 412-413, 2009.

Barabasi, A.-L.: The network takeover, Nat. Phys., 8, 14-16, 2012.

Barthelemy, M.: Spatial networks, Phys. Reports, 499, 1-101, 2011.

Berkowitz, B. and Ewing, R. P.: Percolation theory and network modeling applications in soil physics, Surv. Geophys., 19, 2372, 1998.

Berkowitz, B., Bour, O., and Davy, P.: Odling N.: Scaling of fracture connectivity in geological formations, Geophys. Res. Lett., 27, 2061-2064, 2000.
Bianconi, G. and Barabasi, A.-L.: Competition and multiscaling in evolving networks, Eur. Phys. Lett., 54, 436-442, 2001.

Cárdenas, J. P., Santiago, A., Tarquis, A. M., Losada, J. C., Borondo, F., and Benito, R. M.: Soil porous system as heterogeneous complex network, Geoderma, 160, 13-21, 2010.

Cárdenas, J. P., Santiago, A., Tarquis, A. M., Losada, J. C., Borondo, F., and Benito, R. M.: Community Structure in a Soil Porous System, Soil Sci., 177, 81-97, 2012.

Claussen, J. C.: Offdiagonal Complexity: A computationally quick complexity measure for graphs and networks, Physica A, 375, 365-373, 2007.

Crawford, J. W.: Can complex be simple?, Geoderma, 160, 1-2, 2010.

Dimri, V. P.: Fractal dimension of soil for flow studies, in: Application of fractals in Earth sciences, edited by: Dimri, V. P., A. A. Balkema, 189-193, 2000.

Lal, R.: Soil science and the carbon civilization, Soil Sci. Soc. Am. J., 71, 1425-1437, 2007.

Li, Y., Laidlaw, W. G., and Wardlaw, N. C.: Sensitivity of drainage and imbibition to pore structures as revealed by computer simulation of displacement process, Adv. Colloid Interface Sci., 26, $1-68,1986$.

Masuda, N., Miwa, H., and Konno, N.: Analysis of scale-free networks based on a threshold graph with intrinsic vertex weights, Phys. Rev. E, 70, 036124, doi:10.1103/PhysRevE.70.036124, 2004.

Mooney, S. J. and Korošak, D.: Using Complex Networks to Model Two- and Three-Dimensional Soil Porous Architecture, Soil Sci. Soc. Am. J., 73, 1094-1100, 2009.

Mooney, S. J. and Morris, C.: A morphological approach to understanding preferential flow using image analysis with dye tracers and X-ray Computed Tomography, Catena, 73, 204-211, 2008.

Morita, S.: Crossovers in scale-free networks on geographical space, Phys. Rev. E, 73, 035104-1-035104-4, 2006.

Newman M. E. J.: Assortative mixing in networks, Phys. Rev. Lett., 89, 208701-1-208701-4, 2002.

Perez-Reche, F., Taraskin, S., Neri, F., Gilligan, C., Costa, L. da F., Viana, M., Otten, W., and Grinev, D.: Biological invasion in soil: complex network analysis, in Proc. 16th International Conference on Digital Signal Processing, 2009.

Perez-Reche, F. J., Taraskin, S. N., Otten, W., Viana, M. P., Costa, L. da F., and Gilligan, C. A.: Prominent Effect of Soil Network Heterogeneity on Microbial Invasion, Phys. Rev. Lett., 109, 098102, doi:10.1103/PhysRevLett.109.098102, 2012.

Perrier, E., Tarquis, A. M., and Dathe, A.: A program for fractal and multifractal analysis of two-dimensional binary images: Computer algorithms versus mathematical theory, Geoderma, 134, 284-294, 2006.

Rieu, M. and Sposito, G.: Fractal fragmentation, soil porosity, and soil water properties I. Theory, Soil Sci. Soc. Am. J., 55, 12311238, 1991.

Santiago, A., Cárdenas, J. P., Losada, J. C., Benito, R. M., Tarquis, A. M., and Borondo, F.: Multiscaling of porous soils as heterogeneous complex networks, Nonlin. Processes Geophys., 15, 893902, doi:10.5194/npg-15-893-2008, 2008.

Stanley, E. H. and Meakin, P.: Multifractal phenomena in physics and chemistry, Nature, 335, 405-409, 1988.

Yakubo, K., and Korošak, D.: Scale-free networks embedded in fractal space, Phys. Rev. E, 83, 066111-1-066111-12, 2011. 